A. Berkson and A. McConnell

Nagoya Math. J.

Vol. 49 (1973), 67-75

\title{
LONG EXACT SEQUENCES AND THE TRANSGRESSION RELATION
}

\section{A. BERKSON AND ALAN MCCONNELL}

§1. Introduction. Throughout what follows, let $H$ be a normal subgroup of the group $G, \bar{G}$ be $G / H, M$ a left $G$ module, and $\operatorname{Hom}_{H}(G, M)$ $=\tilde{M}$ a left $G$ module via $(\tau \varphi)(\sigma)=\varphi(\sigma \tau)$

In [1] the present authors compared the 5-term Hochschild Serre sequence to the long exact sequence arising from the short exact sequence of modules $0 \longrightarrow M \stackrel{i}{\longrightarrow} \tilde{M} \stackrel{\pi}{\longrightarrow} \tilde{M} / M \longrightarrow 0$. Since by Shapiro's Lemma, [3, p. 114, Corollary], the following diagram commutes and the vertical map is an isomorphism,

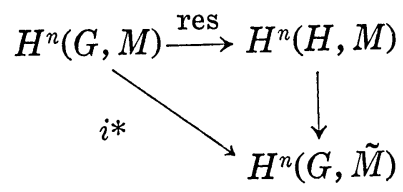

then $H^{n}(G, \tilde{M} / M) \simeq H^{n+1}(G, H ; M)$, i.e. the usual cohomology of pairs [5, Lemma 1.1, p. 230]. In this paper we extend the comparison in [1] to the seven term sequence, and compare these two sequences to a third arising from the short exact sequence of complexes $0 \rightarrow C\left(\bar{G}, M^{H}\right) \rightarrow$ $C(G, M) \rightarrow Q(\bar{G} ; M) \rightarrow 0$. It is also shown that $Q$ is cohomology isomorphic to a subcomplex $\mathscr{D}$ of $C(G, \tilde{M})$. $\mathscr{D}$ consists of transgressive cocycles, and, for each transgressive cocycle class $\bar{x}, \mathscr{D}$ contains at least one distinct $\mathscr{D}$ cocycle class for each element in $t(\bar{x})$ (" $t$ " is transgression); moreover, under the identification of $H^{n}(\mathscr{D})$ with $H^{n}(Q), \delta^{*}(x) \in t(\bar{x})$. Thus one may use transgression as a function in a natural way (§ 2 below).

The principal result of this paper is:

MAIN Theorem If $H^{j}(H, M)=0,0<j<n$, then there are $\mu_{i}$,

Received January 5, 1972. 
$i=1, \cdots, 7$ and $\nu_{i}, i=1, \cdots$ making the following diagram commute. The sixth column is exact, and $\delta^{*} \nu_{3 i}^{-1}$ is the transgression relation.
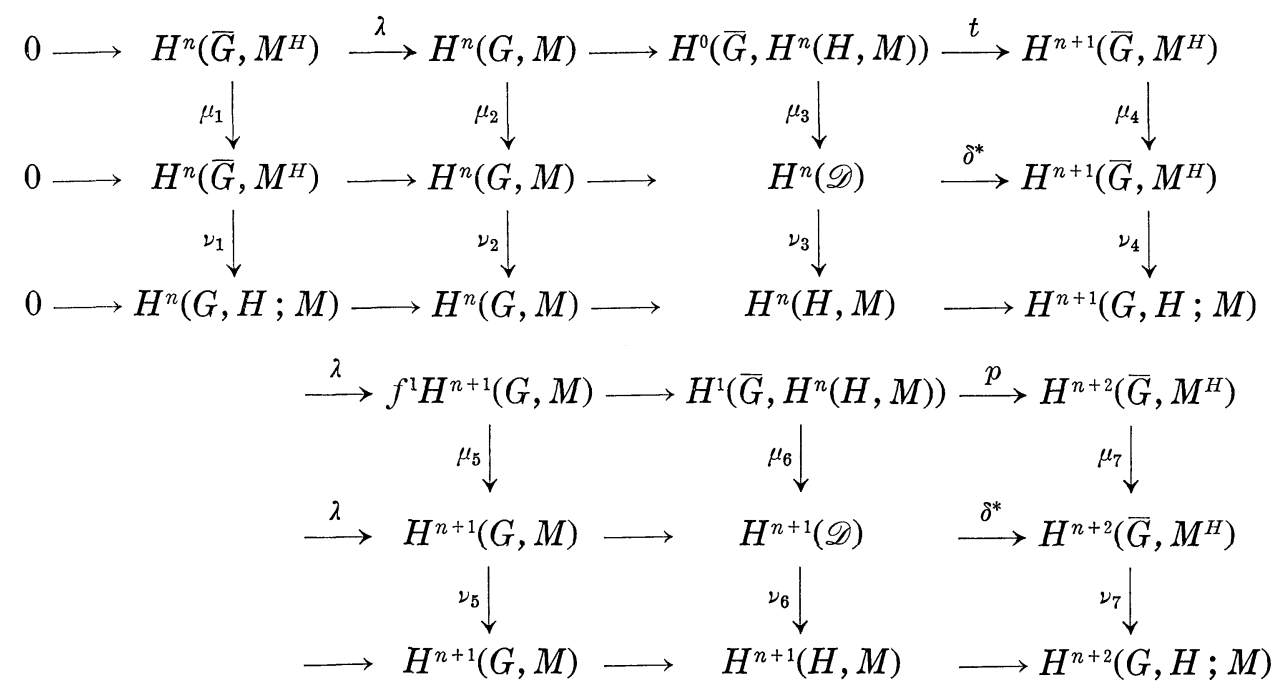

By the above discussion, we can, and shall henceforth, identify $H^{j}(H, M)$ witn $H^{j}(G, \tilde{M})$, and $H^{j}(G, H ; M)$ with $H^{\jmath-1}(G, \tilde{M} / M)$. In the above diagram, the maps $\mu_{1}, \mu_{2}, \mu_{4}, \mu_{7}$, and $\nu_{3 i+2}$ are the appropriate identities, $\mu_{5}$ is inclusion, $\mu_{3}$ and $\nu_{1}$ are isomorphisms, and $\mu_{5}, \mu_{6}, \nu_{3}$ and $\nu_{4}$ are monomorphisms, while $\nu_{3 i}$ are induced by inclusion of complexes under the above identification.

The main theorem extends to Amitsur cohomology as in [1], and the $\mu$ 's can be extended to the Childs sequence [2, Theorem 1, p. 1122] in the obvious way.

It is felt that, in addition to the characterization of the transgressive cocycles, the surprisingly simple description of the largest subcomplex on whose cohomology transgression is a function, is desirable in view of the well known utility of $t$ where it is a function.

Throughout, the following usage will be employed: $\operatorname{Hom}_{H}(A, B)$, where $A$ is a $G$ bimodule and $B$ is a left $G$ module, will be considered a $G$ module by $\left(\tau \varphi(\alpha)=\varphi(\alpha \tau)\right.$, and a $\bar{G}$ module by ${ }^{\sigma} \varphi(\alpha)=\sigma \circ \varphi \circ \sigma^{-1}(a)$. We denote the face maps by $\varepsilon_{i}: \operatorname{Hom}_{G}\left(G^{n}, M\right) \rightarrow \operatorname{Hom}_{G}\left(G^{n+1}, M\right)$, where $\varepsilon_{i} f\left(x_{1}, \cdots, x_{n+1}\right)=f\left(x_{1}, \cdots, \hat{x}_{i}, \cdots, x_{n+1}\right)$. By $C^{n}(G, M)$ we shall mean the homogeneous cochains $\operatorname{Hom}_{G}\left(G^{n+1}, M\right)$ with coboundary operator $d f=$ $\sum_{i=1}^{n+2}(-1)^{i+1}\left(\varepsilon_{i} f\right)$. Functions on $\bar{G}^{i}$ will often be identified with their 
images under inflation.

§ 2. The Second and Third Rows. We start with some general observations on diagrams of complexes; in most cases the proofs are routine and will be omitted. Let

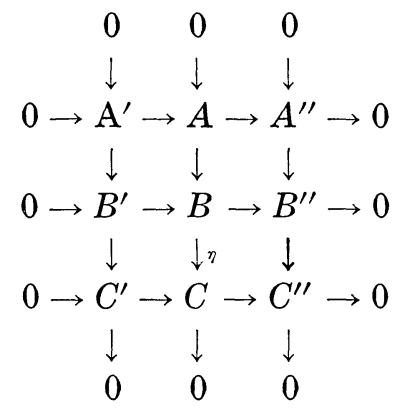

be a commutative diagram of cochain complexes with exact rows and columns. This ensures that $B^{\prime} \cap A=A^{\prime}$ in $B$. Let us assume also that $H^{n}(A)=0$ for all $n>0$. Then $H^{n}\left(A^{\prime \prime}\right) \simeq H^{n+1}\left(A^{\prime}\right)$ and $H^{n}(B) \simeq H^{n}(C)$ for all $n>0$. If we identify $B^{\prime}$ and $A$ with their images in $B$, then $0 \rightarrow B^{\prime}+A \rightarrow B \rightarrow C^{\prime \prime} \rightarrow 0$ is a short exact sequence. Comparing this with the bottom row, we get a map of short exact sequences

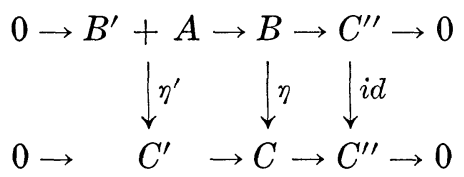

and so a map of long exact sequences of cohomology. By the Five Lemma, $H^{n}\left(B^{\prime}+A\right) \simeq H^{n}\left(C^{\prime}\right)$.

We note that if $b^{\prime}+a \in B^{\prime}+A \subset B$ is a cocycle, then $d b^{\prime}(=-d a$ in $B^{\prime} \cap A=A^{\prime}$ ) is a cocycle of $A^{\prime}$. From the first column of (I) we obtain a long exact sequence $\cdots \rightarrow H^{n}\left(A^{\prime}\right) \rightarrow H^{n}\left(B^{\prime}\right) \rightarrow H^{n}\left(C^{\prime}\right) \rightarrow \cdots$. Since we want to replace $H^{n}\left(C^{\prime}\right)$ by $H^{n}\left(B^{\prime}+A\right)$, we observe that if $H^{n}\left(B^{\prime}+A\right)$ $\rightarrow H^{n+1}\left(A^{\prime}\right)$ is defined by $\operatorname{cl}\left(b^{\prime}+a\right) \rightarrow \operatorname{cl}\left(d b^{\prime}\right)$ then the following commutes:

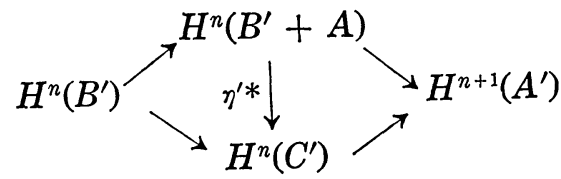

Thus we have a new long exact sequence $\cdots \rightarrow H^{n}\left(A^{\prime}\right) \rightarrow H^{n}\left(B^{\prime}\right) \rightarrow$ 
$H^{n}\left(A+B^{\prime}\right) \rightarrow H^{n+1}\left(A^{\prime}\right) \rightarrow \cdots$. Since $H^{n}\left(A^{\prime \prime}\right) \simeq H^{n+1}\left(A^{\prime}\right)$, we get a map $H^{n+1}\left(A^{\prime}\right) \rightarrow H^{n}\left(B^{\prime \prime}\right)$. Thus

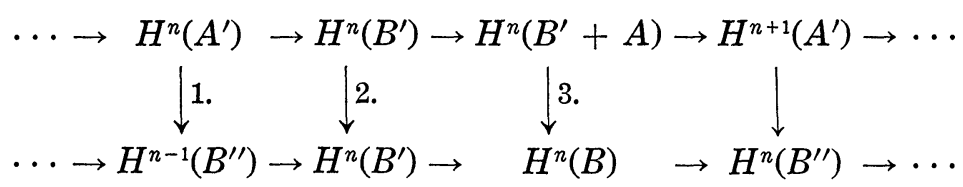

is commutative is squares 1 . and 2., anticommutative in square 3 . If for $H^{n}\left(B^{\prime}+A\right) \rightarrow H^{n+1}\left(A^{\prime}\right)$, we had chosen $c l\left(b^{\prime}+a\right) \rightarrow c l(d a)$, square 3 would commute. We have more or less arbitrarily chosen to put the anti-commutativity here.

We apply the above to the following diagram II.

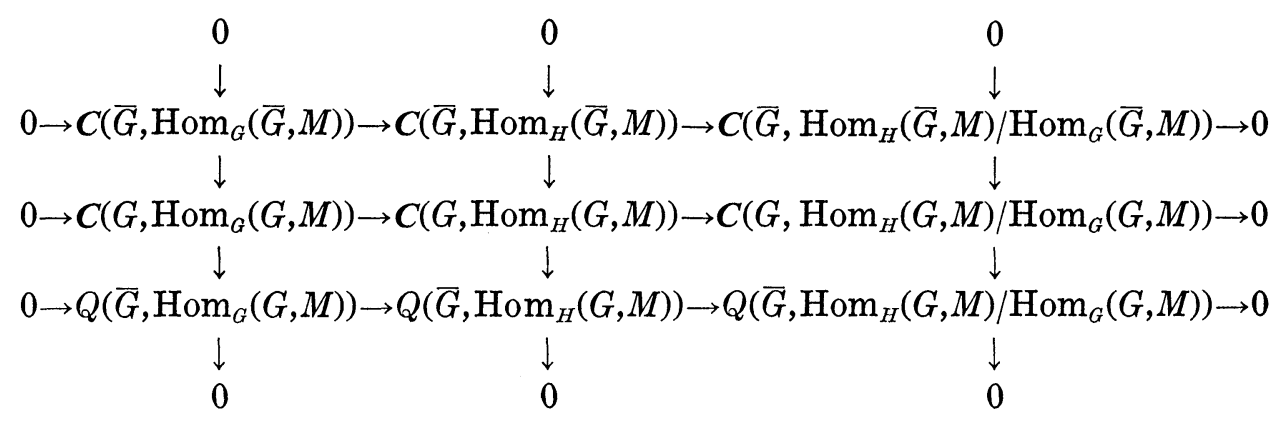

In (II) all coefficient modules have right multiplication on the domain as their module structure for both $G$ and $\bar{G}$.

We note that $\operatorname{Hom}_{G}(\bar{G}, M) \simeq M^{H} ; \operatorname{Hom}_{H}(\bar{G}, M) \simeq \operatorname{Hom}_{H}\left(G, M^{H}\right) \simeq$ $\operatorname{Hom}\left(\bar{G}, M^{H}\right) ; \operatorname{Hom}_{G}(G, M) \simeq M ;$ and $\operatorname{Hom}_{H}\left(G, M^{H}\right) \cap \operatorname{Hom}_{G}\left(G, M^{H}\right) \simeq M^{H}$. Hence it is easily seen that elements $u \in C(G, M)$ and $v \in C\left(\bar{G}, \operatorname{Hom}\left(\bar{G}, M^{H}\right)\right)$ have the same image in $C(G, \tilde{M})$ iff they are images of the same element in $C\left(\bar{G}, M^{H}\right)$. This pull-back property in the upper left hand square of (II) guarantees that (II) is a $3 \times 3$ commutative diagram of complexes with exact rows and columns. Also, since $\operatorname{Hom}\left(\bar{G}, M^{H}\right)$ is coinduced [6, p. 120], $H^{n}\left(\bar{G}, \operatorname{Hom}\left(\bar{G}, M^{H}\right)\right)=0$. Thus (II) satisfies the hypothesis of (I) and we record our results.

TheOREM I. Let $\mathscr{D}=C\left(\bar{G}, \operatorname{Hom}\left(\bar{G}, M^{H}\right)\right)+C(G, M)$ be identified with its image in $C(G, \tilde{M})$. Then there are maps which make the following diagram commutative with exact rows. 


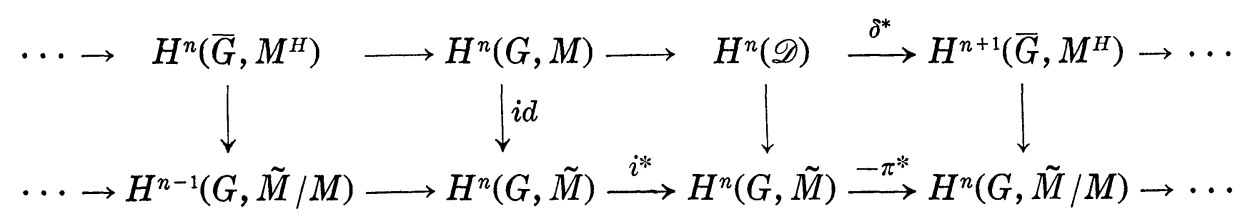

The sign of $\pi^{*}$ has been changed to get rid of the anticommutativity. The map from $H^{n}(\mathscr{D}) \rightarrow H^{n}(G, \tilde{M})$ is induced by the inclusion of complexes.

THEOREM II. The relation $\delta^{*} \nu_{3 i}^{-1}$ is exactly the transgression relation [3, p. 129].

Proof: Routine verification.

§3. The Spectral Sequence. In order to establish the notation and setting for the remainder of this paper, it is necessary to recapitulate in the first two paragraphs of this section some of the classical results of Hochschild and Serre [3]. Let $E_{0}^{m, n}=\operatorname{Hom}_{E}\left(\bar{G}^{m+1} \times G^{n+1}, M\right)$, which implies that $E_{0}^{m, n} \subset E_{0}^{m-1, n+1}$. Define ' $d: E_{0}^{m, n} \rightarrow E_{0}^{m+1, n}$ and " $d: E_{0}^{m, n} \rightarrow$ $E_{0}^{m, n+1}$ by

$$
' d f=\sum_{i=1}^{m+2}(-1)^{i+1} \varepsilon_{i} f, \quad \quad \prime d f=\sum_{i=m+2}^{n+2}(-1)^{i+1} \varepsilon_{i} f .
$$

We have " $d ' d+{ }^{\prime} d \prime d=0, "{ }^{\prime \prime} d=0$ and ' $d$ ' $d=0$; this defines a double complex and an associated single complex Tot, where $\operatorname{Tot}^{n}=\sum_{i+j=n} E_{0}^{i, j}$. Tot can be filtered by rows or columns, giving rise two spectral sequences ' $E_{r}$ and " $E_{r}$. We now observe that each row has a contracting homotopy $s: E_{0}^{m, n} \rightarrow E_{0}^{m-1, n}(n \geqq 0, m>0)$ given by $(s f)\left(x_{1}, \cdots, x_{m}\right.$, $\left.y_{1}, \cdots, y_{n+1}\right)=f\left(x_{1}, \cdots, x_{m}, \bar{y}_{1}, y_{1}, \cdots, y_{n+1}\right)$; hence $E_{1}^{m, n}=0$ for $m>0$, $n \geqq 0$, and the second spectral sequence collapses. Whence $H^{n}(T o t) \simeq$ ${ }^{\prime \prime} E_{2}^{0, n}$. Furthermore, the kernel of ' $d: E_{0}^{0, n} \rightarrow E_{0}^{1, n}$ can be identified with $\operatorname{Hom}_{G}\left(G^{n+1}, M\right)$ so that ${ }^{\prime \prime} E_{2}^{0, n} \simeq H^{n}(G, M)$. Thus the chain of inclusions $C(G, M) \rightarrow E_{0}^{0, n} \rightarrow$ Tot induces an isomorphism of cohomology $H^{n}(G, M) \rightarrow$ $H^{n}(T o t)$.

Next, one computes ' $E_{2}^{m, n}$. Since $E_{0}^{m, n}$ may be identified with $\operatorname{Hom}_{\bar{G}}\left(\bar{G}^{m+1}, \operatorname{Hom}_{H}\left(G^{n+1}, M\right)\right)$, and $\operatorname{Hom}_{\bar{G}}\left(\bar{G}^{m+1},-\right)$ is an exact functor, one has ${ }^{\prime} E_{2}^{m, n} \simeq H^{m}\left(\bar{G}, H^{n}(H, M)\right)$. In particular, $H^{0}(H, M)=M^{H}$ implies that ${ }^{\prime} E_{2}^{m, 0}=H^{m}\left(\bar{G}, M^{H}\right)$.

Following an idea of Pareigis-Rosenberg [4], we define a cochain 
$\operatorname{map} \phi: T o t \rightarrow C(G, M)$ which induces the isomorphism $H^{n}(T o t) \rightarrow H^{n}(G, M)$ inverse to the isomorphism induced by the inclusion of complexes. First we define $\theta^{m, n}: E_{0}^{m, n} \rightarrow C^{m+n}(G, M)$ by $\left(\theta^{m, n} f\right)\left(x_{1}, \cdots, x_{m+n+1}\right)=$ $(-1)^{m} f\left(x_{1}, \cdots, x_{m+1}, x_{m+1}, \cdots, x_{m+n+1}\right)$. A routine computation shows that for $f \in E_{0}^{m, n}$, the following formula holds:

$$
\theta^{m+1, n} d f+\theta^{m, n+1 \prime \prime} d f=d \theta^{m, n} f .
$$

We then set $\phi\left(\left(f_{0, m+n} ; \cdots ; f_{m+n, 0}\right)\right)=\theta^{0, m+n} f_{0, m+n}+\cdots+\theta^{m+n, 0} f_{m+n, 0}$. It is immediate from (III) that $\phi$ is a cochain map. Since $C(G, M) \longrightarrow$ $E_{0}^{0, n} \longrightarrow T o t \stackrel{\phi}{\longrightarrow} C(G, M)$ is the identity map, $\phi$ induces the isomorphism. We remark that if $f \in E_{0}^{m, n}$, then $\left(\theta^{m, n} f\right)$ depends only on the cosets $\bmod H$ of its first $m$ variables.

Now we impose the hypothesis $H^{i}(H, M)=0$ for $0<i<n$. This ensures that ' $E_{2}^{m, i}=0$ for $0<i<n$. A standard spectral sequence argument shows that there is an exact sequence

$' E_{2}^{0, n-1}=0 \rightarrow^{\prime} E_{2}^{n, 0} \rightarrow H^{n}(T o t) \rightarrow^{\prime} E_{2}^{0, n} \rightarrow^{\prime} E_{2}^{n+1,0} \rightarrow f^{1} H^{n+1}(T o t) \rightarrow{ }^{\prime} E_{2}^{1, n} \rightarrow^{\prime} E_{2}^{n+2,0}$

Here $f^{1} H^{n+1}(T o t)$ is the first filtration group of the filtered total cohomology group. Using the identification discussed above, we get the standard 7-term fundamental sequence in the first row of the main theorem.

THEOREM III. There exist maps $\mu_{i}, i=1, \cdots, 7$ which make the squares of the first two rows of the diagram in the main theorem commute, and which have the properties required, where $\mu_{1}, \mu_{2}, \mu_{4}$, and $\mu_{7}$ are the identities and $\mu_{5}$ is inclusion.

Proof. The problem is to define $\mu_{3}$ and $\mu_{6}$. We shall define and carry out the commutativity argument for $\mu_{6}$ (the case of $\mu_{3}$ is analogous but somewhat easier and will be omitted). The notation established here will be used later.

Let $A \in E^{\prime} E_{2}^{1, n} ; \alpha_{1, n} \in A, \alpha_{1, n} \in E_{0}^{1, n}$. This means that " $d \alpha_{1, n}=0$, and $' d \alpha_{1, n} \in E_{0}^{2, n}$ is the image under " $d$ of $-\alpha_{2, n-1}$ in $E_{0}^{2, n-1}$. Exploiting the vanishing of the vertical cohomology in the double complex for $0<i<n$, we get a sequence of elements $\alpha_{i, n+1-i}, \quad(i=1, \cdots, n)$ such that $' d \alpha_{i, n+1-i}+{ }^{\prime \prime} d \alpha_{i+1, n-i}=0$ (see [1, p. 408] for a more through discussion of this process). Finally set ' $d \alpha_{n+1,0}=(-1)^{n} \gamma \in E_{0}^{n+2,0}$.

Obviously ${ }^{\prime} d \gamma=0= \pm\left(\varepsilon_{n+4} \gamma-\varepsilon_{n+5} \gamma\right)$. This says that $\gamma \in \operatorname{Hom}_{G}\left(\bar{G}^{n+3} \times G, M\right)$ 
is independent of the last variable. We shall identify $\gamma$ with $\beta \in \operatorname{Hom}_{G}\left(\bar{G}^{n+3}, M\right) \simeq \operatorname{Hom}_{\bar{G}}\left(\bar{G}^{n+3}, M^{H}\right) . \quad \beta$ is clearly an $n+2$ cocycle in $C\left(\bar{G}, M^{H}\right)$; indeed $\operatorname{cl}(\beta)=p(A)$ in $H^{n+2}\left(\bar{G}, M^{H}\right)$. Also, $\theta^{n+2,0} \gamma\left(x_{1}, \cdots, x_{n+3}\right)$ $=(-1)^{n+2} \gamma\left(x_{1}, \cdots, x_{n+3}, x_{n+3}\right)=(-1)^{n+2} \beta\left(x_{1}, \cdots, x_{n+3}\right)$.

For the $n+1$ cochain $z=\left(0 ; \alpha_{1, n} ; \cdots ; \alpha_{n+1,0}\right) \in \operatorname{Tot}^{n+1}$, we have $d_{\text {Tot }}(z)=\left(0 ; \cdots ;(-1)^{n} \gamma\right) \in \operatorname{Tot}^{n+2}$. Hence $f=\phi(z)$ has coboundary $d f=\phi\left(d_{T o t}(z)\right)=\beta$. Let $\quad \tilde{\mu}_{6}: H^{\prime}\left(\bar{G}, H^{n}(H, M)\right) \rightarrow H^{n+1}(Q)$ be defined by $A \rightarrow \operatorname{cl}(f)$.

We now construct explicitly the isomorphism $\psi: H^{m}(Q) \rightarrow H^{m}(\mathscr{D})$ inverse to the given projection $\eta^{\prime}$. This will give us the formula for $\mu_{6}(A)$ which we will need in the next section. If $\operatorname{cl}(\alpha) \in H^{m}(Q)$, i.e. $\alpha \in C^{m}(G, M)$ with $d \alpha$ in $C^{n+1}\left(\bar{G}, M^{H}\right)$, then let $\psi$ be given by

$$
\begin{aligned}
& \psi(\alpha)\left(\sigma_{1}, \cdots, \sigma_{m+1}\right)(\tau) \\
&=\sum_{i=1}^{m+1}(-1)^{i+m} \tau \alpha\left(\tau^{-1}, \sigma_{1}, \cdots, \hat{\sigma}_{i}, \cdots, \sigma_{m+1}\right) \\
&= \tau \alpha\left(\sigma_{1}, \cdots, \sigma_{m+1}\right)+(-1)^{m} \tau d \alpha\left(\tau^{-1}, \sigma_{1}, \cdots, \sigma_{m+1}\right) \in C^{m}\left(G, \operatorname{Hom}_{G}(G, M)\right) \\
&+C^{m}\left(\bar{G}, \operatorname{Hom}\left(\bar{G}, M^{H}\right)=\mathscr{D} .\right.
\end{aligned}
$$

Since $\psi \eta^{\prime}(a+b)=\psi(a)=a+b^{\prime}$, it follows that $\psi \eta^{\prime}(a+b)-(a+b)$ $=b^{\prime}-b$, a cocycle in $H^{m}\left(\bar{G}, \operatorname{Hom}\left(\bar{G}, M^{H}\right)=0\right.$. Therefore $b^{\prime}-b=d \varphi$ is a coboundary in $\mathscr{D}$ and $\psi \eta^{\prime}$ is the identity on $H^{m}(\mathscr{D})$. Since we know $\eta^{\prime}$ is an isomorphism, $\psi$ must be a two sided inverse.

We can now define $\mu_{6}(A)=\psi\left(\tilde{\mu}_{6} A\right)$, for if we vary our choices of the $\alpha_{i, n+1-i}$, we change them by a coboundary in Tot. Since $\phi$ is a cochain map and $\psi$ is an isomorphism of cohomology, $\mu_{6}$ is well defined.

Next we show that square 6 is commutative. As was pointed out, $p(A)=\operatorname{cl}(\beta)$. But the definition of $H^{n+1}(\mathscr{D}) \rightarrow H^{n+2}\left(\bar{G}, M^{H}\right)$ sends $\operatorname{cl}(\psi(f))$ to $\operatorname{cl}(d f)=\operatorname{cl}(\beta)$. It is to be noted here that if we had chosen the sign of $H^{n}(\mathscr{D}) \rightarrow H^{n+1}\left(\bar{G}, M^{H}\right)$ differently, this square would anticommute. It was remarked previously that we put the anticommutativity elsewhere.

To complete the proof, we show that square 5 is commutative. Let $\operatorname{cl}(\alpha) \in f^{\prime} H^{n+1}(G, M)$ where $\alpha \in C^{n+1}(G, M)$; the image of $\operatorname{cl}(\alpha)$ in $H^{n+1}(\mathscr{D})$ is $\operatorname{cl}(\alpha+0)$. By virtue of the definition of $f^{\prime} H^{n+1}(G, M)$, there is a cocycle $z=\left(0 ; \alpha_{1, n} ; \cdots ; \alpha_{n+1,0}\right) \in \operatorname{Tot}^{n+1}, \operatorname{cl}(\phi(z))=\operatorname{cl}(\alpha)$. Finally, the image of $\operatorname{cl}(\alpha)$ under $f^{\prime} H^{n+1}(G, M) \rightarrow E_{2}^{1, n}$ is $\operatorname{cl}\left(\operatorname{cl}\left(\alpha_{1, n}\right)\right)$. But $\mu_{6}\left(\operatorname{cl}\left(\operatorname{cl}\left(\alpha_{1, n}\right)\right)\right)$ is easily seen to be $\operatorname{cl}(\alpha+0)$. 
§4. Exactness of the Sixth Column. Given $A$ in $H^{1}\left(\bar{G}, H^{n}(H, M)\right)$, take $z=\left(0 ; \alpha_{1, n} ; \cdots ; \alpha_{0, n+1}\right)$ as in the definition of $\mu_{6}$. Since all the terms depend only on the $H$-coset of at least the first two variables, $\phi(z)=f$ depends only on the $H$-coset of its first variable. Let $u\left(\sigma_{1}, \cdots, \sigma_{n+1}\right)(\tau)=\tau f\left(\tau^{-1}, \sigma_{1}, \cdots, \sigma_{n+1}\right)$. Then $u \in C^{n}(G, \tilde{M})$. Now we have $d u\left(\sigma_{1}, \cdots, \sigma_{n+2}\right)(\tau)=\sum_{i=1}^{n+2}(-1)^{i+1} \tau f\left(\tau^{-1}, \sigma_{1}, \cdots, \hat{\sigma}_{i}, \cdots, \sigma_{n+2}\right)=(-1)^{n} \psi(f)=$ $(-1)^{n} \mu_{6}(A)$. Hence $d m \mu_{6} \subset \operatorname{Ker} \nu_{6}$.

To show that $\operatorname{Im} \mu_{6} \supset \operatorname{Ker} \nu_{6}$, we need to $\operatorname{map} C^{m}(G, \tilde{M})$ into the double complex by sending $\varphi \rightarrow K(\varphi)$ where $K(\varphi)\left(\bar{\sigma}, \sigma_{1}, \cdots, \sigma_{m+1}\right)=$ $\tilde{\sigma} \varphi\left(\sigma_{1}, \cdots, \sigma_{m+1}\right)\left(\bar{\sigma}^{-1}\right)$. We observe that $K(d \varphi)=-" d K \varphi$, and that for $c l(x) \in H^{m}(Q), K \psi(x)=(-1)^{m}\left(d x-\varepsilon_{1} x\right)$.

If $B \in H^{n+1}(\mathscr{D}), \nu_{6}(B)=0, B=\psi(C)$, and $c \in C$, then, in $C^{n+1}(G, \tilde{M})$, $\nu_{6} \psi(c)=d u$. Let $\alpha_{1, n}={ }^{\prime} d K u+(-1)^{n+1} d c$, where $d c \in \operatorname{Hom}_{\bar{G}}\left(\bar{G}^{n+3}, M^{H}\right) \subset$ $\mathrm{Hom}_{G}\left(\bar{G}^{i+1} \times G^{n+2-i}, M\right)$. Then

$$
\begin{aligned}
{ }^{\prime \prime} d \alpha_{1, n} & =-^{\prime} d^{\prime \prime} d K u+(-1)^{n+1 \prime \prime} d d c={ }^{\prime} d K d w+(-1)^{n \prime} d(d c) \\
& =' d\left(K d w+(-1)^{n}\left(d c-\varepsilon_{1} c\right)\right)={ }^{\prime} d(K d w-K \psi(c))=0 .
\end{aligned}
$$

Also, ' $d \alpha_{1, n}=(-1)^{n+1 \prime} d(d c)=(-1)^{n \prime \prime} d(d c)$. Hence $c l\left(\alpha_{1, n}\right) \in H^{\prime}\left(\bar{G}, H^{n}(H, M)\right)$. One verifies that $\tilde{\mu}_{6}\left(c l\left(\alpha_{1, n}\right)\right)=\theta^{1, n} d K u=-\theta^{0, n+1 \prime \prime} d K u=\theta^{0, n+1}(K d w)=$ $\theta^{0, n+1}(K \psi(c))=(-1)^{n+1} \theta^{0, n+1}\left(d c-\varepsilon_{1} c\right)=(-1)^{n+1} c$, and consequently we have that $\mu_{6}\left(c l\left(\alpha_{1, n}\right)\right)=(-1)^{n+1} \psi(c)=(-1)^{n+1} B$, proving

THEOREM 4. The sixth column in the main theorem is exact.

The proof of Theorem 4 proves exactly.

Proposition The sequence $E_{m}^{1, m-1} \rightarrow H^{m}(\mathscr{D}) \rightarrow H^{m}(H, M)$ is exact. Since $\mu$ is a cochain map, we may consider $E_{m}^{1, m-1}$ to be in $E_{2}^{1, m-1}=$ $H^{1}\left(\bar{G}, H^{m-1}(H, M)\right)$. This gives us

COROLlaRy If $H^{1}\left(\bar{G}, H^{m-1}(H, M)\right)=0$, transgression is single valued.

COROLlaRy If $H^{1}\left(\bar{G}, H^{n-2}(H, M)\right)=H^{n-1}(H, M)=H^{n}(H, M)=0$ then $H^{n}\left(\bar{G}, M^{H}\right) \simeq H^{n}(G, M)$. If $H^{n}(H, M)=0, n \geq k$, then $H^{n}\left(G / H, M^{H}\right) \simeq$ $H^{n}(G, M)$ for $n \geqq k+2$ and $H^{k+1}\left(G / H, M^{H}\right) \rightarrow H^{k+1}(G, M) \rightarrow 0$ is exact.

\section{BIBLIOGRAPHY}

[1] A. J. Berkson and Alan McConnell, On inflation-restriction exact sequences in group and Amitsur cohomology, Trans. Amer. Math. Soc., 141 (1969), 403-413.

[2] L. N. Childs, The exact sequence of low degree and normal algebras, Bull. Amer. 
Math. Soc., 76 (1970), 1121-1124.

[ 3 ] G. Hochschild and J.-P. Serre, Cohomology of group extensions, Trans. Amer. Math. Soc., 74 (1953), 110-134.

[4] B. Pareigis and A. Rosenberg, Adendum to "Amitsur's complex for inseparable fields," Osaka J. Math., 1 (1964), 33-44.

[5] L. Ribes, On a cohomology theory for pairs of groups, Proc. Amer. Math. Soc., 21 (1969), 230-234.

[6] J.-P. Serre, Corps Locaux, Hermann, Paris, 1962.

University of Illinois

Howard University 